\title{
WI - Call for Papers Heft 3/2011
}

\section{Nachhaltiges Cloud-Computing}

DOI 10.1007/s11576-009-0205-7

\section{Herausgeber des Schwerpunktthemas}

\author{
Prof. Dr. Günter Müller \\ Albert-Ludwigs-Universität Freiburg \\ Friedrichstr. 50 \\ 79098 Freiburg \\ Deutschland \\ mueller@iig.uni-freiburg.de
}

\author{
Prof. Dr. Noboru Sonehara \\ Prof. Dr. Isao Echizen \\ Dr. Sven Wohlgemuth \\ National Institute of Informatics \\ 2-1-2 Hitotsubashi \\ Chiyoda-ku \\ Tokyo 101-8430 \\ Japan \\ sonehara@nii.ac.jp \\ iechizen@nii.ac.jp \\ wohlgemuth@nii.ac.jp
}

This article is also available in English via http://www.springerlink.com and http://www.bise-journal.org: Müller G, Sonehara N, Echizen I, Wohlgemuth S (2010) BISE - Call for Papers, Issue 3/2011, Sustainable Cloud Computing. Bus Inf Syst Eng. doi: 10.1007/ s12599-009-0083-y.

\section{Schwerpunktthema}

Nach Mainframe und Client-ServerComputing ist Cloud-Computing das nächste Paradigma der elektronischen Datenverarbeitung. Cloud-Computing ist die moderne Version des TimeSharing-Modells der sechziger Jahre; allerdings mit dem wesentlichen Unterschied, dass Privatnutzer und Unternehmen die Dienste der „Cloud des Internets" mit einem Webbrowser nutzen sowie sich Rechenleistung und Datenspeicher der Cloud teilen. Die Herausgabe persönlicher Daten und digitaler Inhalte von Nutzern einer Cloud und die damit zugleich verbundene Anhäufung dieser Daten bei Dienstleistern einer Cloud vereinfachen in dieser massiv gemeinsam genutzten Infrastruktur ihre zusätzliche Nutzung, z. B. ihre Auswertung durch Dritte. Erste Anwendungen der fortgeschrittenen und weltweit durchgeführten Weitergabe und sekundären Nutzung persönlicher Daten und digitaler Inhalte an Dritte zeigen die folgenden Probleme auf, die sich nachteilig auf die sich für den wirtschaftlichen Einsatz bietenden Chancen des Cloud-Computing auswirken:

(a) Rechtliche Normierungen, wie bspw. SOX, HIPAA und nationale Datenschutzgesetze, können die Nutzung der Cloud für bestimmte Anwendungen verhindern. So schränkt die europäische Datenschutzdirektive die Weitergabe von persönlichen Daten an Dienstleister in Drittländer ein. Ein Lösungsansatz ist die Anwendung strikter Normen, der allerdings die Möglichkeiten des CloudComputing verringert und dessen Nutzungskosten erhöhen könnte.

(b) Dienstleister einer Cloud bieten oftmals keine Dienstleistungsverträge (Service Level Agreements) an, obwohl die Verfügbarkeit der Daten aus Sicht der Nutzer einer Cloud eine zwingende Voraussetzung ist. Unternehmen würden Cloud-Computing nicht für ihre kritischen Anwendungen nutzen, wenn die Verfügbarkeit und Verlässlichkeit der Dienste einer Cloud nicht garantiert sind.

(c) Obwohl Privatnutzer und Unternehmen ihre IT-Systeme mit Firewalls und Intrusion-Detection-Systemen schützen können, ist der Schutz ihrer Geschäftsprozesse und Daten vor den Dienstleistern einer Cloud nicht möglich. Eine Cloud ist eine Blackbox: Sie abstrahiert von den Sicherheitsprozessen als auch von den Orten der Datenspeicherung. Die Nutzer müssen den Dienstleistern einer Cloud vertrauen, dass sie persönliche Daten und digitale Inhalte entsprechend nach den rechtlichen
Normierungen und vereinbarten Regeln verarbeiten.

Elektronische Patientenakten sind ein Beispiel. Sie dienen der Ansammlung medizinischer Daten, um deren Verfügbarkeit und Vollständigkeit zu verbessern. Dies hat eine Effizienzverbesserung der Geschäftsprozesse medizinischer Dienstleistungen zur Folge und unterstützt die sekundäre Nutzung medizinischer Daten z. B. für statistische Zwecke. Da elektronische Patientenakten nicht an einzelne medizinische Einrichtungen gebunden sind, kann deren Verwaltung von Dienstleistern angeboten werden, die über das notwendige Wissen und die Erfahrung für den Betrieb geeigneter Datenbanken verfügen. Rechtliche Normierungen wie bspw. das Gesetz zur Modernisierung der gesetzlichen Krankenversicherung (GKV-Modernisierungsgesetz - GMG) in Deutschland und das USamerikanische Gesetz zur „Health Insurance Portability and Accountability“ (HIPPA) verbieten i. Allg. jegliche Weitergabe identifizierender medizinischer Daten an Dritte, sofern die ausdrückliche Einwilligung des Patienten nicht vorliegt. Bestehende Systeme für elektronische Patientenakten sind mit diesen rechtlichen Normen konform, indem sie die Patienten über die Nutzung und Weitergabe ihrer Daten entscheiden lassen. Allerdings werden vier wesentliche Schutzmaßnahmen nicht erfüllt: Erstens garantieren sie nicht die Verlässlichkeit ihres Dienstes und die Verfügbarkeit der elektronischen Patientenakten. Zweitens bieten sie keine Mechanismen an, mit denen die Patienten die Einhaltung der rechtlichen Normen sowie die Durchsetzung ihrer Entscheidungen durch die Dienstleister überprüfen können. Drittens können Patienten nach einer genehmigten Weitergabe Auflagen bezüglich der weitergehenden Nutzung und Weitergabe ihrer Daten durch Dritte weder anmelden noch durchsetzen. Viertens garantieren die Dienste nicht die Vertraulichkeit der medizinischen Daten gegenüber dem Dienstleister. Dieser sollte 
keinen Zugriff auf die medizinischen Daten der Patienten haben, da andernfalls das Risiko einer unbefugten Veröffentlichung und Weitergabe vergrößert wird.

Das Schwerpunktthema „Nachhaltiges Cloud-Computing" soll neue Methoden, Technologien und Best Practices der Industrie zur Lösung der Probleme in den Bereichen Verlässlichkeit, Compliance und Datenschutz für Cloud-Computing sammeln. Die Artikel sollen zum nachhaltigen Cloud-Computing trotz unterschiedlicher Regulierungs- und Vertrauensbereiche der Cloud beitragen.

Beiträge aus Forschung und Praxis sind u. a. zu den folgenden (gerne auch weiteren) Themenfeldern erwünscht:

- De-Perimeterization und IT-Risikomanagement für Cloud-Computing

- Zuverlässige und sichere Interaktion zwischen Diensten

- Authentifizierung und Autorisierung für Cloud-Computing

- Private Information Processing und Multi-Party-Computation

- Privacy-enhancing Technologies für die sekundäre Nutzung von persönlichen Daten

- IT-Compliance und Datenschutzstrategien für Cloud-Computing unter Berücksichtigung unterschiedlicher rechtlicher Normierungen
- Best Practices der De-Perimeterization für Cloud-Computing

- Ökonomie und Cloud-Computing

\section{Einreichung von Beiträgen}

Bitte reichen Sie Beiträge für die $\mathrm{Ru}$ briken WI - Aufsatz und WI - State of the Art bis spätestens 2010-07-01 über das Online-Begutachtungssystem (http://www.editorialmanager.com/ buis/) ein. Bitte beachten Sie die Hinweise zu formaler Gestaltung und Umfang von Beiträgen für die WIRTSCHAFTSINFORMATIK/Business \& Information Systems Engineering (BISE). Vollständige Beiträge sollten höchstens zehn Druckseiten umfassen; das entspricht 50.000 Zeichen einschließlich Leerzeichen, abzüglich 5.000 Zeichen je Seite an Bildern. Ausführliche Autorenrichtlinien stehen unter http://www.wirtschaftsinformatik. de zum Download bereit.

Eingereichte Beiträge werden anonymisiert von mehreren Gutachtern in einem doppelt-blinden Verfahren auf Relevanz, Originalität und fachliche Qualität beurteilt. Neben den Herausgebern des Schwerpunktthemas und jenen der Zeitschrift wirken dabei weitere ausgewiesene internationale Persönlichkeiten aus Wissenschaft und Praxis mit.

Ergänzend zu den Aufsätzen sind auch weitere Einreichungen zum Schwerpunktthema willkommen.

Angenommene Beiträge erscheinen identisch in Deutsch und Englisch. Die deutschsprachige Fassung erscheint in WIRTSCHAFTSINFORMATIK, die englischsprachige in Business \& Information Systems Engineering (BISE). Angenommene Beiträge werden in enger Zusammenarbeit von Autoren und einem professionellen Übersetzerteam übersetzt.

\section{Zeitplan}

Einreichung von Beiträgen: 2010-07-01

Benachrichtigung der Autoren: 2010-08-26

Abschluss der ersten Überarbeitung: 2010-20-28

Benachrichtigung der Autoren:

2010-12-26

Ggf. Abschluss einer zweiten Überarbeitung (einsprachig): 2011-01-20

Ggf. Abschluss einer zweiten Überarbeitung (zweisprachig): 2011-02-17

Geplanter Erscheinungstermin

Heft 3/2011: Juni 2011. 